\title{
The Price Forecasting of Military Aircraft Based on SVR
}

\author{
Jifeng Tong1, Jiaxing Du1, Ping Chen², Jianguang Yuan², Zhan Huan² \\ ${ }^{1}$ The Ministry of Science Research, Academy of Armored Force Engineering, Beijing, China \\ ${ }^{2}$ Institute of Nonlinear Science, Academy of Armored Force Engineering, Beijing, China \\ Email: dht12004@163.com, qingpingguo 135@163.com
}

Received January 2015

\begin{abstract}
The difficulty of the prediction of military aircraft purchase price lies in the small sample data, and the sample data have the complicated non-linear characteristics. By analyzing the influence of parameters of aircraft purchase price, SVR is proposed to predict the aircraft purchasing price model, and uses the model to predict the aircraft purchase price. The calculation results show that the prediction of the purchase price to establish military aircraft model has higher prediction accuracy.
\end{abstract}

\section{Keywords}

Military Aircraft, SVR, The Purchase Price

\section{Introduction}

With the increasing requirement of modern warfare, military aircrafts are increasingly using high technology, new processes and new materials, leading to a sharp rise in the development and production costs, the purchase price is also rising, and the contradiction between lack of defense expenditure is becoming increasingly acute, which makes the prediction of purchase price becoming more and more important. Prediction of military aircraft purchase price has become an important content of military aircraft. But with the development of science and technology, it makes the system performance and complexity of modern military aircraft constantly increasing. There are many factors to influence the aircraft purchase prices, and it also put forward higher requirements for the military aircraft procurement price prediction models. It needs to study more to put forward more accurately forecast model of the proposed military aircraft purchase price

Statistical learning theory (SLT) is a machine learning rule of a specialized research in small samples under the theory established by Vapnik. Support vector machine (SVM) is developed on the basis of this theory into a new classification and regression tools. Support vector regression is mainly including $\varepsilon$-SVR presented by Vapnik and $v$-SVR proposed by Schlkopf [1] [2]. $\varepsilon$-SVR control algorithm is hoping to achieve precision by predetermined $\varepsilon$, and $v$-SVR to minimize , so as to ensure that the algorithm can achieve the highest precision. Support vector machine to improve the generalization ability through the structural risk minimization principle, solves the small sample, non-linear, high dimension, local minimum and so on, it has been widely used in [3]-[6]. Aiming at the existing problems of the characteristics of few sample data and support vector regression prediction of military aircraft purchase price, we provide a method to predict the military aircraft purchase price 
based on SVR.

\section{The Basic Principle of v-SVR Support Vector Regression}

A training set

$$
T=\left\{\left(x_{1}, y_{2}\right), \cdots\left(x_{l}, y_{l}\right)\right\} \in(X \times Y)^{l},
$$

among them $x_{i} \in X=R^{n}, y_{i} \in Y=R, i=1,2, \cdots, l$, which uses the nonlinear mapping of $\varphi(x)$ the input vector $x$ is mapped to the feature space, and then linear regression in high dimensional feature space, construct the regression function

$$
f(x)=\omega^{T} \cdot \varphi(x)+b
$$

Among them $\omega$ and $b$ respectively denote the weight vector and bias. The introduction of slack variables $\xi^{(*)}=\left(\xi_{1}, \xi_{1}^{*}, \cdots, \xi_{l}, \xi_{l}^{*}\right)^{T}$ and the penalty parameter $C$, and to construct the two times planning original problem:

$$
\begin{gathered}
\min _{\omega \in R^{n}, \xi^{(*)} \in R^{2 l}, b \in R} \frac{1}{2}\|\omega\|^{2}+C \cdot\left(v \varepsilon+\frac{1}{l} \sum_{i=1}^{l}\left(\xi_{i}+\xi_{i}^{*}\right)\right) \\
\text { s.t. } \quad\left(\omega^{T} x_{i}+b\right)-y_{i} \leq \varepsilon+\xi_{i}, \quad i=1,2, \cdots, l \\
y_{i}-\left(\omega^{T} x_{i}+b\right) \leq \varepsilon+\xi_{i}^{*}, \quad i=1,2, \cdots, l \\
\varepsilon, \xi_{i}, \xi_{i}^{*} \geq 0, \quad i=1,2, \cdots, l
\end{gathered}
$$

Type (2) for the dual problem:

$$
\begin{gathered}
\min _{\alpha^{(*)} \in R^{2 l}} \frac{1}{2} \sum_{i, j=1}^{l}\left(\alpha_{i}^{*}-\alpha_{i}\right)\left(\alpha_{j}^{*}-\alpha_{j}\right) K\left(x_{i}, x_{j}\right)-y_{i} \sum_{i=1}^{l}\left(\alpha_{i}^{*}-\alpha_{i}\right) \\
\text { s.t. } \quad \sum_{i=1}^{l}\left(\alpha_{i}^{*}-\alpha_{i}\right)=0 \\
0 \leq \alpha_{i}^{*}, \alpha_{i} \leq \frac{C}{l}, i=1,2, \cdots, l \\
\sum_{i=1}^{l}\left(\alpha_{i}^{*}-\alpha_{i}\right) \leq C \cdot v
\end{gathered}
$$

$\alpha, \alpha^{*}$ is the Lagrange multiplier, $K\left(x_{i}, x_{j}\right)$ as a satisfying Mercer condition symmetric kernel function, kernel function are commonly used polynomial kernel function (Polynomial), radial basis function (RBF), Sigmoid kernel function etc.

v-SVR algorithm steps:

1) A training set

$$
T=\left\{\left(x_{1}, y_{2}\right), \cdots\left(x_{l}, y_{l}\right)\right\} \in(X \times Y)^{l},
$$

The

$$
x_{i} \in X=R^{n}, y_{i} \in Y=R, i=1,2, \cdots, l
$$

2) Select the appropriate positive $v, C$, and kernel function $K\left(x, x^{\prime}\right)$.

3) Constructing and solving the optimization problem (3), to obtain the optimal solution

$$
\bar{\alpha}=\left(\bar{\alpha}_{1}, \bar{\alpha}_{1}^{*}, \cdots, \bar{\alpha}_{l}, \bar{\alpha}_{l}^{*}\right)^{T}
$$

4) To construct the decision-making function

$$
f(x)=\sum_{i=1}^{l}\left(\bar{\alpha}_{i}^{*}-\bar{\alpha}_{i}\right) K\left(x_{i}, x\right)+\bar{b}
$$


The choice is located in the open interval in $\bar{\alpha}_{j}$ or $\bar{\alpha}_{k}^{*}$.

Order:

$$
\bar{b}=\frac{1}{2}\left[y_{i}+y_{k}-\left(\sum_{i=1}^{l}\left(\bar{\alpha}_{i}^{*}-\bar{\alpha}_{i}\right) K\left(x_{i}, x_{j}\right)+\sum_{i=1}^{l}\left(\bar{\alpha}_{i}^{*}-\bar{\alpha}_{i}\right) K\left(x_{i}, x_{k}\right)\right.\right.
$$

\section{Military Aircraft Price Prediction}

Now, military turbofan transporter purchase price prediction model is established to analysis as an example. A lot of parameters to describe the performance of turbofan transporter, then we take 8 typical examples of feature parameters, which include: the flat maximum take-off weight is $x_{1}$, fuselage length is $x_{2}$, the height of the plan is $x_{3}$, take off distance is $x_{4}$, the voyage rang with full oil is $x_{5}$, the optimal height of oil fly speed is $x_{6}$, aircraft empty weight is $x_{7}$ and maximum fuel load is $x_{8}$. The price indicated by $y$, the benchmark price for the year 2004.

The 9 type of turbofan transporter sample performance parameters and purchasing prices listed in Table 1. In order to carry out the error analysis and prediction test of the model, we select the 8 sub sample table as training samples, I models for testing samples.

We take the maximum take-off weight, body length, and maximum height of the plane, the take-off distance, full range, the optimal height of oil fly speed, aircraft empty weight and maximum fuel capacity as the input parameters, we take the price as output, kernel function takes the radial basis kernel function (RBF)

$$
K\left(x, x^{\prime}\right)=\exp \left(-\gamma\left\|x-x^{\prime}\right\|^{2}\right),
$$

Among them, $\gamma=0.5$, the penalty coefficient $C=1, v=0.5, \varepsilon=0.001$, we establish $v$-SVR model, Comparison between the measured value and fitted value as shown in Table 2 . As can be seen, SVR model to fit the average value of relative error is only $5.37 \%$, the coefficient of determination is $R^{2}=0.99$, and the fitting effect is good.

To predict the price of I transport plane by this model, get the forecasting results as shown in Table 3.

Table 1. Performance parameters and acquisition costs of transporters samples.

\begin{tabular}{cccccccccc}
\hline Type & $x_{1} / \mathrm{kg}$ & $x_{2} / \mathrm{m}$ & $x_{3} / \mathrm{m}$ & $x_{4} / \mathrm{m}$ & $x_{5} / \mathrm{km}$ & $x_{6} / \mathrm{m} \cdot \mathrm{s}^{-1}$ & $x_{7} / \mathrm{kg}$ & $x_{8} / \mathrm{kg}$ & $x_{9} /$ Million Yuan \\
\hline$A$ & 13494 & 23.500 & 8.43 & 867 & 4262 & 425.0 & 425.0 & 5683 & 6666.70 \\
$B$ & 6849 & 14.390 & 4.57 & 987 & 3701 & 746.0 & 746.0 & 2640 & 3624.30 \\
$C$ & 9979 & 16.900 & 5.12 & 1581 & 4679 & 874.0 & 874.0 & 3350 & 6569.90 \\
$D$ & 5670 & 13.340 & 4.57 & 536 & 3641 & 536.0 & 536.0 & 1653 & 5586.23 \\
$E$ & 63503 & 39.750 & 9.30 & 1859 & 6764 & 925.0 & 925.0 & 21273 & 27768.80 \\
$F$ & 22000 & 29.870 & 6.75 & 1200 & 2870 & 907.0 & 907.0 & 5500 & 17575.20 \\
$G$ & 21500 & 27.170 & 7.65 & 1050 & 2000 & 580.0 & 580.0 & 5000 & 18137.60 \\
$H$ & 70310 & 29.790 & 11.66 & 1091 & 7876 & 602.0 & 602.0 & 36300 & 50476.00 \\
$I$ & 21000 & 24.615 & 7.30 & 1300 & 3100 & 819.2 & 819.2 & 6000 & 14250.00 \\
\hline
\end{tabular}

Table 2. Comparison between the measured value and fitted value of transport price.

\begin{tabular}{cccc}
\hline Type & The observation values & The fitted values & The relative error \\
\hline A & 6666.7 & 7140.14 & 7.10 \\
B & 3624.3 & 4072.22 & 12.36 \\
C & 6569.9 & 7017.82 & 6.82 \\
D & 5586.23 & 6075.55 & 8.76 \\
E & 27768.8 & 27292.88 & -1.71 \\
F & 17575.2 & 18057.98 & 2.75 \\
G & 18137.6 & 17671.77 & -2.57 \\
H & 50476 & 50013.42 & -0.92 \\
\hline
\end{tabular}


Table 3. The prediction price results of transport plane.

\begin{tabular}{cccc}
\hline type & The measured value & The fitted values & The relative error \\
\hline I & 14250.00 & 14173.95 & -0.53 \\
\hline
\end{tabular}

The practical results show: SVR has stronger generalization ability in the case of limited samples, SVR has certain universality, it can be used as a suitable method and it should be popularized.

\section{Conclusion}

A small sample of multivariate data is a difficult problem to predict the military aircraft in the purchase price, and support vector regression is a new statistical learning model by the principle of structural risk minimization instead of empirical risk minimization principle, and it has perfect theory basis. Based on the analysis of the price data, using support vector regression theory, we establish the model of aircraft purchase price. From the example above we can see that, the method of support vector machine have a better calculation accuracy, and stronger generalization ability in dealing with nonlinear problems.

\section{References}

[1] Vapnik, V N. (1999) An Overview of Statistical Learning Theory. IEEE Trans. on Neural Network, 10, 988-999. http://dx.doi.org/10.1109/72.788640

[2] Schlkopf, B., et al. (2000) New Support Vector Algorithms. Neural Computation, 12, 1207-1245. http://dx.doi.org/10.1162/089976600300015565

[3] Chen, Q., Wu, M.C., Xue, Y.J., Yang, J.F. and Liu, G.Y. (2009) Carbon Flux Based on Support Vector Regression Prediction. Computer Engineering and Applications, 45, 235-238. (In Chinese)

[4] Li, D.Q. (2009) Application of Support Vector Machine Regression Method in Prediction of Ship's Price Index. Ship \& Ocean Engineering, 2, 104-106. (In Chinese)

[5] Lian, C.B., Zhao, Y.J., Li, H.L., Qu, F., Cai, F.L. and Zhang, J.T. (2008) Based on Support Vector Machine Regression Prediction of Coalbed Gas Content. J. Xi'an University of Science and Technology, 28, 707-710. (In Chinese)

[6] Li, R. and Li, G.M. (2008) Predict the Output of Photovoltaic Power Generation Based on Support Vector Machine Regression. China Electric Power, 41, 74-78. (In Chinese) 